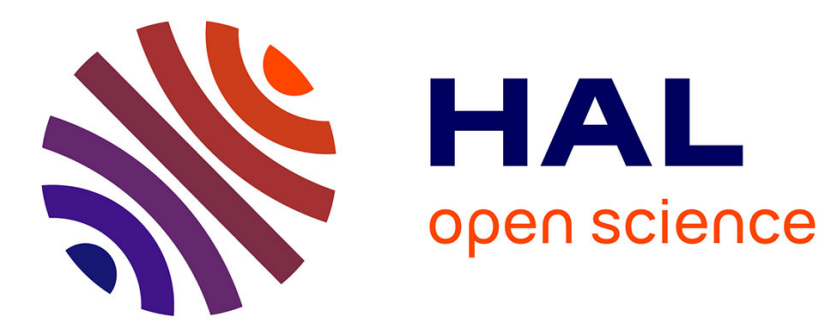

\title{
Dialogue sud-atlantique dans la favela: mise en récit de l'immigration angolaise par une compagnie de théâtre brésilienne
}

Nicolas Quirion

\section{- To cite this version:}

Nicolas Quirion. Dialogue sud-atlantique dans la favela: mise en récit de l'immigration angolaise par une compagnie de théâtre brésilienne. Lusotopie, 2021, 19 (2), pp.213-234. 10.1163/1768308412341761 . hal-03336161

\section{HAL Id: hal-03336161 \\ https://hal.science/hal-03336161}

Submitted on 7 Sep 2021

HAL is a multi-disciplinary open access archive for the deposit and dissemination of scientific research documents, whether they are published or not. The documents may come from teaching and research institutions in France or abroad, or from public or private research centers.
L'archive ouverte pluridisciplinaire HAL, est destinée au dépôt et à la diffusion de documents scientifiques de niveau recherche, publiés ou non, émanant des établissements d'enseignement et de recherche français ou étrangers, des laboratoires publics ou privés. 


\title{
Dialogue sud-atlantique dans la favela : mise en récit de l'immigration angolaise par une compagnie de théâtre brésilienne
}

\author{
Nicolas Quirion \\ Unité de Recherche Migrations et Société, Université de Paris \\ Instituto de Pesquisa e Planejamento Urbano e Regional, Université Fédérale de Rio de \\ Janeiro \\ nicoquirion@gmail.com
}

\section{Résumé}

Par l'observation d'une création théâtrale, Hoje não saio daqui, le présent travail cherche à examiner les relations entre habitants brésiliens des favelas et immigrés angolais au sein du quartier de Maré, à Rio de Janeiro. Certaines questions soulevées par le spectacle sont complétées par des données ethnographiques collectées sur le terrain, afin de proposer une réflexion ayant trait aux relations entre l'Angola et le Brésil, deux pays de langue portugaise liés par l'Atlantique Sud.

\section{Mots-clés}

Angola - Brésil - migration - favela - Atlantique sud

Diálogo sul-Atlântico na favela : narrativas da imigração angolana por uma companhia de teatro brasileira.

\section{Resumo}

Pela observação de uma criação teatral ("Hoje não saio daqui"), o presente trabalho procura indagar as relações entre moradores brasileiros das favelas e imigrantes 
angolanos no bairro de Maré, no Rio de Janeiro. Algumas questões levantadas pelo espetáculo são confrontadas com dados etnográficos coletados no campo, isso a fim de propor uma reflexão sobre as relações entre Angola e Brasil, dois países de língua portuguesa ligados pelo Atlântico Sul.

\title{
Palavras-Chave
}

Angola - Brasil - migração - favela - Atlântico Sul

South Atlantic dialogue in the favela: Angolan immigration narrated by a Brazilian theatre company

\begin{abstract}
This study seeks to examine relations between Brazilian inhabitants of favelas and Angolan immigrants in the Maré district of Rio de Janeiro through the performance of a theatrical creation, Hoje não saio daqui. Some of the questions raised in the play relate to ethnographic data from fieldwork to explore the nature of relations between Angola and Brazil, two Portuguese-speaking countries connected by the South Atlantic Ocean.
\end{abstract}

\section{Keywords}

Angola - Brazil - migration - favela - South Atlantic 


\section{Introduction}

Situé dans le secteur nord de la municipalité de Rio de Janeiro, le "Complexo da Maré $^{l}$ " est un très vaste groupement de favelas et d'ensembles sociaux dégradés qui compte environ 140000 habitants. Depuis la seconde moitié des années 1990, certaines localités de ce quartier ${ }^{2}$ accueillent une population d'immigrés angolais réputée relativement importante. Cette situation a fréquemment suscité la curiosité d'observateurs externes; en effet, la concentration durable d'étrangers au sein d'un quartier pauvre est peu habituelle dans le Brésil contemporain. On aurait par ailleurs pu présupposer que la démographie des favelas de Rio de Janeiro (dont la majorité de la population est noire ${ }^{3}$ ) ainsi que la pratique d'une langue commune ${ }^{4}$ permettraient aux immigrants angolais de progressivement se "fondre dans la masse". Toutefois, comme nous le verrons par la suite, plusieurs facteurs ont concouru à l'essentialisation du groupe au sein même de la favela, tout en lui conférant à l'extérieur une notoriété disproportionnée au regard de sa véritable importance numérique. C'est ainsi que, dans l'imaginaire carioca ${ }^{5}$, de nos jours encore, l'Angolais représente souvent l'idéal-type de l'Africain immigré, une figure de l'altérité parfois entourée de représentations négatives et de peurs.

Fin 2019, au cours d'un travail de terrain réalisé dans le cadre d'une recherche doctorale $^{6}$, j'ai pu accompagner quelques répétitions ainsi qu'assister à deux représentations de la nouvelle création d'un groupe de théâtre basé à Maré : la "Cia Marginal". Active depuis 2005 et proche d'une importante $\mathrm{ONG}^{7}$ œuvrant en faveur du

\footnotetext{
${ }^{1}$ Selon la catégorisation proposée en 2010 par le Secretaria Municipal de Habitação e Cidadania (SMHC) de Rio de Janeiro, les ensembles de favelas qui forment une tâche urbaine unique, sont considérées comme des "complexes" ("complexos") de favelas.

2 Le territoire du "bairro" (quartier) de Maré, qui comprend 16 favelas et ensembles résidentiels populaires, a été délimité officiellement pour la première fois par un décret municipal en 1988.

3 Le "Censo populacional da Maré" (2018) établit que 62,1\% des habitants du quartier de Maré appartiendraient à la catégorie censitaire "Negro" (conçue comme la jonction des catégories "Pardo" e "Preto").

${ }^{4}$ Pour une discussion approfondie autour du concept de "lusophonie" et de son usage politique, voir notamment Cahen \& Dos Santos (2018).

${ }_{6}^{5}$ Relatif à la ville de Rio de Janeiro

6 Dans ce cadre, plus ample, il s'agissait d'observer les conflits, mais également les relations de convivialité qui se tissent à partir de l'implantation d'immigrants internationaux d'origines diverses au sein de certains quartiers informels de la ville de Rio de Janeiro. L'un des terrains de cette enquête, basée sur plus de quatre années d'observations participantes, se situe justement à Maré.

${ }^{7}$ Il s'agit de l'ONG REDES da Maré < https://www.redesdamare.org.br> Consulté le 21/09/2020.
} 
développement de la citoyenneté dans la favela, la compagnie en question se positionne politiquement en déclarant être formée de "jeunes activistes, qui cherchent à transformer

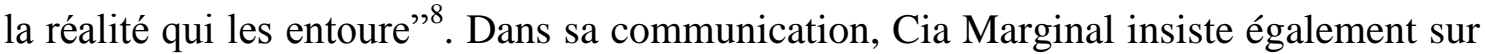
le fait que ses acteurs résident presque tous à Maré, et sont en majorité noirs. Ceux-ci sont par ailleurs présentés comme co-créateurs des pièces dans lesquelles ils se produisent.

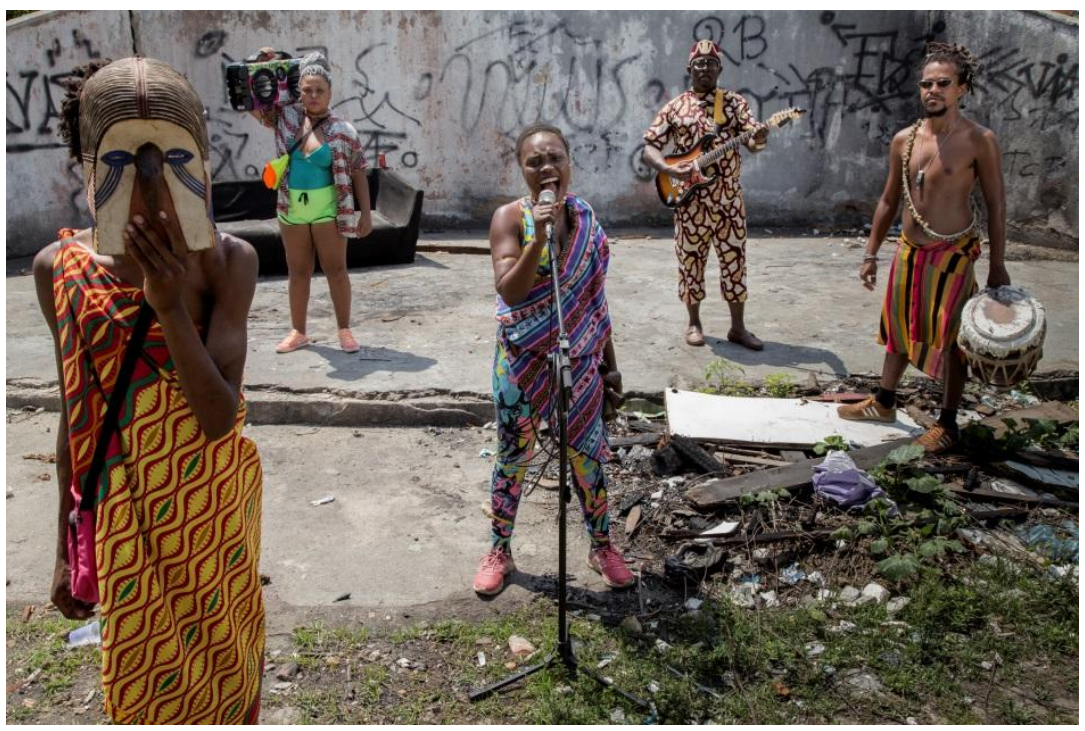

Figure 1: illustration accompagnant le dossier de presse du spectacle (Gê Vasconcelos, 2019)

L'œuvre dont il sera question ici — qui porte pour titre "Hoje não saio daqui" — a fortement retenu mon attention. En effet, ce spectacle cherche à se saisir de l'expérience migratoire d'Africains dans la favela afin de rendre particulièrement visibles certaines questions ayant trait à des discriminations pouvant atteindre une population bien plus large. Lors d'un entretien qu'elle m'a accordé, Isabel Penoni, directrice de la compagnie, a ainsi déclaré que la pièce était née d'une intention de "penser l'intensification de la xénophobie et du racisme dans le monde, la difficulté dans la relation avec l'autre". Or, d'après elle, la troupe souhaitait "traiter ces thèmes à partir de la communauté angolaise de Maré”. Il fut ainsi décidé de recruter cinq acteurs angolais ainsi qu'un directeur musical ango-congolais. Ceux-ci apparaissent de fait dans la pièce, aux côtés des intégrants habituels de la compagnie, et ont également contribué à l'élaboration de la mise en scène.

Comme indiquent les mots employés par la responsable de la compagnie, nous verrons qu'à Maré, le groupe angolais est fréquemment perçu comme formant une

\footnotetext{
${ }^{8}$ <https://pt-br.facebook.com/ciamarginal> Consulté le 21/09/2020.
} 
“communauté" au sein de la favela. Pour les habitants, il s'agirait en outre d'un groupe qui se caractérise non seulement par certains identificateurs ethniques (peau plus foncée que la plupart des "Noirs" brésiliens, code vestimentaire particulier, etc.), mais également par des pratiques et un tempérament réputés différents du reste de la population locale.

Le présent article ne constitue pas une critique de la pièce "Hoje não saio daqui". Il s'agit plutôt de se saisir de certains points mis en exergue par le spectacle afin de les inscrire dans un contexte plus général. Ceci nous amène à formuler quelques questions autour desquelles tournera notre réflexion : sur quelles bases s'établissent les relations entre des Brésiliens marginalisés et une population immigrée originaire d'un pays comme l'Angola ? Quels liens ou ruptures entre les deux pays sont mis en évidence par les difficultés et dilemmes spécifiques rencontrés par des Angolais au Brésil — et plus spécifiquement au sein de la favela où un nombre significatif d'entre eux a établi résidence? Enfin, quelle contribution à l'historique relation sud-atlantique (Alencastro 1986) peut être suggérée par l'expérience migratoire angolaise à Maré, ici mise en récit de façon théâtrale et militante?

Afin d'aborder ces questions, nous analyserons le déroulé de la pièce, en nous concentrant plus particulièrement sur une scène (dite de "la Bataille") au cours de laquelle les acteurs brésiliens et angolais confrontent les représentations que chaque groupe a construites sur l'autre au sein de la favela. Les situations dramaturgiques seront également rapprochées d'éléments empiriques collectés lors de l'enquête de terrain menée à Maré. Mais, pour commencer, afin de mieux appréhender le sujet dont traite la pièce de théâtre, nous caractériserons brièvement l'espace urbain en question ; puis, nous donnerons quelques éléments concernant l'implantation angolaise qui s'y est développée.

\section{Maré, un territoire complexe}

Le quartier aujourd'hui connu comme Maré correspondait jadis à une vaste zone humide, de marais et de mangrove, le long de la baie de Guanabara. L'occupation informelle de la région débuta dans les années 1940, et s'accéléra dans les décennies suivantes en raison de l'expansion des activités industrielles vers le nord de la ville, comme en témoignent les nombreux entrepôts (souvent abandonnés) qui bordent la favela. Ainsi, la longue frange marécageuse se peupla progressivement de travailleurs 
pauvres, généralement originaires de la région Nord-Est du Brésil. Les demeures autoconstruites sur pilotis, reliées entre elles par de fragiles passerelles, y représentèrent durant des décennies la forme emblématique d'habitat (Diniz 2013). Mais la précarité de ces bicoques, trop visibles le long des voies express qui relient l'aéroport au centreville, était embarrassante pour les pouvoirs publics. De nombreuses interventions urbaines eurent ainsi lieu dans la région, incluant différentes phases de remblaiement ainsi que la construction de logements sociaux. Initialement projetés par l'État, ceux-ci se dégradèrent et se transformèrent rapidement, laissant place à un vaste ensemble de favelas (Jacques et al 2002).

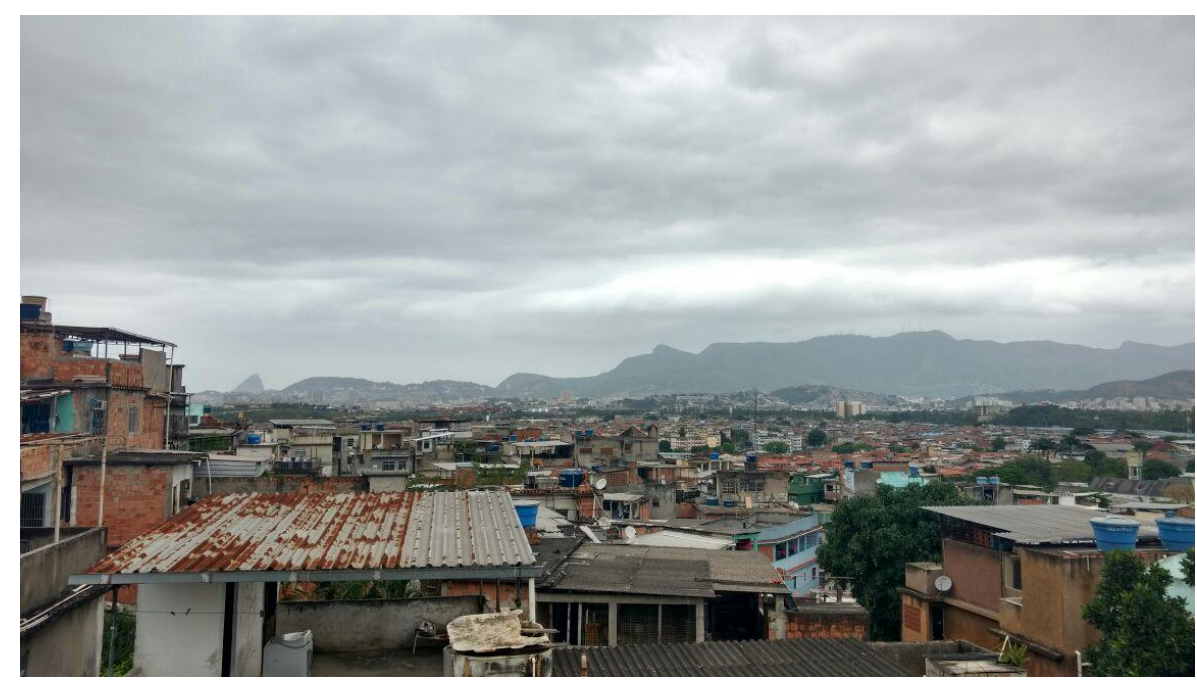

Figure 2 : Aperçu du Complexe de Maré (Photo de l'auteur, 2016)

De nos jours, Maré possède une réputation extrêmement contrastée. D’un côté, la presse et les autorités locales ne cessent de dépeindre ses favelas comme une sorte de centre névralgique du crime organisé au sein de la ville. Il est vrai que les conflits armés entre groupes de narcotrafiquants rivaux, ainsi qu'entre ceux-ci et la police, y sont particulièrement réguliers et violents. Mais, Maré est également connue pour son historique de mobilisations populaires (Leite 2013), la vigueur actuelle de sa société civile ainsi que l'intensité de ses manifestations culturelles et artistiques. Le dynamisme créatif local est notamment favorisé par l'existence d'équipements culturels dans une proportion bien plus importante qu'à l'accoutumée dans les favelas de Rio. Souvent administrés par des ONG, des musées et salles de spectacle ont vu le jour dans des entrepôts désaffectés, aux frontières entre la ville formelle et les favelas, suggérant ainsi la formation d'un "nouveau territoire culturel” (Vaz 2010) où s'exprimerait la richesse créative des groupes sociaux marginalisés. 


\section{La présence angolaise à Maré}

Luiz Felipe de Alencastro (1986) a documenté avec une grande précision les relations économiques qui se tissèrent entre différents ports brésiliens et celui de Luanda (Angola), à partir du XVI siècle. La majeure partie des personnes déportées d'Afrique pour être réduites à l'esclavage au Brésil était originaire de la région aujourd'hui délimitée en tant que République d'Angola. L'interdiction du trafic négrier transatlantique, en 1850, provoqua néanmoins une interruption nette des flux humains entre ces deux rives de l'Atlantique Sud.

À une échelle et pour des motifs profondément différents, l'immigration angolaise représente toutefois, depuis les années 1970, le principal flux de personnes en provenance d'Afrique vers le Brésil. L'importance relative de cette présence peut s'expliquer de façon très générale par une communauté linguistique, ainsi que par le développement de complexes dynamiques diplomatiques, culturelles et industrialocommerciales entre les deux nations au cours des 45 dernières années (Muñoz 2016).

Cependant, la nature et l'importance de l'immigration angolaise vers le Brésil ont beaucoup varié au gré des évènements qui agitèrent le pays d'Afrique subsaharienne (Aydos 2010). La phase qui nous intéresse correspond au mouvement migratoire le plus important, qui se produisit au cours des années 1990 et jusqu'au début des années 2000, lorsqu'une population relativement importante de jeunes hommes fuyant les "rusgas" (recrutement militaire obligatoire) s'établit, préférentiellement, dans la ville de Rio de Janeiro. Généralement issue des classes populaires et anciens résidents des "musseques" (quartiers populaires de Luanda), une grande partie de ces individus se concentra progressivement dans une série de favelas appartenant au quartier de Maré. Cette implantation résidentielle fut principalement motivée par le coût moindre de l'hébergement, par la proximité avec l'aéroport international (qui facilitait la pratique de la “mukunza", le commerce transnational informel), ainsi que par la formation progressive d'un réseau de solidarité entre compatriotes (Petrus 2001). Cependant, ces étrangers furent objets de rumeurs et suspicions formulées par la police, les médias, ainsi que certains élus locaux. Une piste d'enquête policière (jamais éclaircie, mais massivement divulguée) soupçonnait en effet certains Angolais de former les trafiquants de drogue locaux aux techniques de guérilla urbaine (Quirion 2018). De plus, les immigrés étaient victimes de diverses discriminations au sein même de la favela, où une 
réputation de stellionataires les suivait (Santana 2016). Ainsi, dans l'imaginaire collectif, de nos jours encore, la figure de l'immigré angolais est bien souvent associée à des représentations négatives englobantes.

L'importance numérique de la population angolaise à Maré a en outre fait l'objet de nombreuses spéculations à ses origines. Si la très forte mobilité des migrants de première génération a pu rendre toute estimation difficile, de nos jours cette présence semble s'être stabilisée à un niveau bas. Un recensement mené récemment par une organisation locale (Redes da Mare 2018 : 32-33) a avancé que, même si les Angolais représentent bien le seul groupe étranger bénéficiant d'une présence remarquable sur le territoire, à peine 195 individus vivraient au sein des favelas de Maré ${ }^{9}$. Parmi eux, 151 hommes et seulement 43 femmes. Le profil de ces étrangers dans la favela a en outre fortement évolué depuis l'époque pionnière, en raison d'une sensible mobilité économique ascendante et de l'arrivée d'individus davantage éduqués et de catégories sociales légèrement plus élevées.

Bien qu'aujourd'hui limitée à un petit nombre d'individus réparti sur l'ensemble du quartier, l'implantation migratoire à Maré se distingue par le fonctionnement de lieux de sociabilité (bars, restaurants et autres commerces) administrés et fréquentés, principalement, par des Angolais. Au cœur de la favela Vila do Pinheiro, une intersection de rues localement connue sous le nom de "esquina dos angolanos" s'est consolidée comme point de rencontre entre compatriotes. Il est important de noter que des immigrés de même nationalité, venus d'autres quartiers ou même d'autres régions, fréquentent également ces lieux, occasionnant ainsi un flux régulier de personnes externes qui ajoute à la visibilité du groupe dans la favela.

\footnotetext{
${ }^{9}$ Les organisateurs de l'étude signalent néanmoins, pertinemment, que ce chiffre peut être sous-estimé en raison d'une crainte des individus à se déclarer étrangers, cela "en raison d'une éventuelle illégalité du processus migratoire".
} 


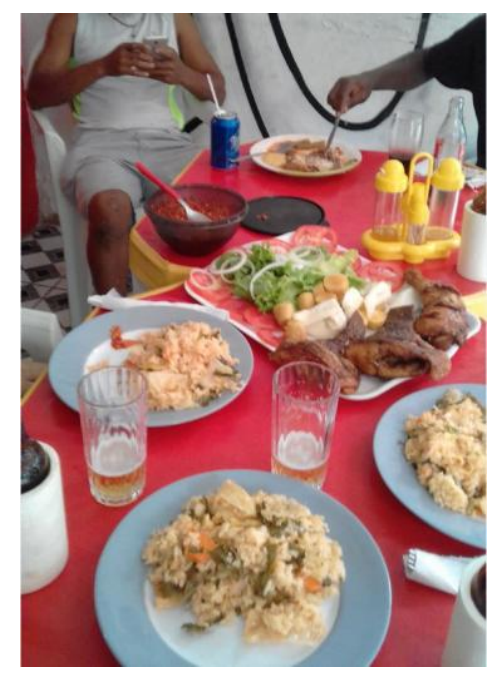

Figure 3 : "Mufete", spécialité angolaise servie dans un restaurant de Maré (Photo de l'auteur, 2018)

Les relations sociales observées à Maré jusqu'à nos jours montrent que les immigrés angolais, anciens ou récents, représentent un groupe social considéré à part, dont la couleur (généralement plus foncée que celle des Noirs brésiliens) s'ajoute à d'autres signes distinctifs (accent, emploi d'un argot spécifique, etc.) pour produire un régime d'altérité. Si de nombreuses interactions et une convivialité limitée peuvent être observées entre le reste des habitants de la favela et les Angolais, l'entre-soi reste important chez ces derniers. Ce repli peut correspondre aussi bien à des affinités propres au groupe que répondre à un certain rejet.

Les déclarations et le profil de Nzaje ${ }^{10}, 21$ ans, l'un des acteurs de la pièce de théâtre sont, à ce titre, particulièrement intéressants. Nzaje est né en Angola mais est arrivé au Brésil avec ses parents en 1999, alors âgé de deux ans. L'importance de la préservation culturelle chez les Angolais apparait fortement dans son discours :

“À la maison, la culture a toujours été africaine. Au plus je pourrai garder cette culture en moi, au mieux ce sera. Au-delà de la porte, il y a le Brésil ; mais à l'intérieur, c'est autre chose. À la maison, on parle avec l'accent africain au moins $50 \%$ du temps."

Par ailleurs, si les propos de Nzaje mettent en évidence son caractère profondément biculturel (puisqu'élevé au Brésil), ils indiquent également une

\footnotetext{
10 J'ai rédigé un portrait de ce jeune artiste pour une revue communautaire distribuée à Maré <https://mareonline.com.br/arteecultura/cultura/nzaje-a-cultura-como-refugio/> Consulté le 21/09/2020.
} 
segmentation des cercles sociaux assez habituelle chez ses compatriotes présents à Maré :

“Le truc, c'est que je sépare beaucoup. J'ai mes amis angolais et brésiliens. (...) Même si ça peut être chouette d'être ensemble, on dirait qu'il y a toujours des petits conflits de culture qui apparaissent. C'est pas des engueulades, mais quelqu'un finit toujours par dire: "C'est bizarre ça, bizarre..." Et donc, toi, tu dois expliquer : "Non, c’est qu'au Brésil ça se passe comme ça, mais en Angola c'est comme ci"."

\section{Aperçu du spectacle}

Si l'appropriation et la reconversion à visées culturelles d'installations industrielles constituent un trait marquant à Maré (Vaz 2010), la pièce dont il sera ici question prend pour scène l'un des seuls espaces verts existant au sein de l'ensemble de favelas : le "Parque Ecológico Municipal da Maré". Cette petite colline couverte de végétation était jadis une île, avant qu'une vaste opération de remblaiement de la baie de Guanabara ne la rattache au continent dans les années 1980.

Deux cycles de représentations de la pièce "Hoje não saio daqui" eurent lieu : du 7 au 22 décembre 2019, puis du 24 janvier au 9 février 2020. Le spectacle fut offert sous la forme d'une déambulation au sein du "Parque Ecológico". D'une durée de plus de deux heures, la pièce se décompose en trois mouvements, qui correspondent aux déplacements physiques effectués par les acteurs et le public entre trois différents points du parc. L'espace naturel ainsi que celui de la favela alentour sont amplement utilisés comme supports dramatiques. Pour déclencher le premier mouvement et ainsi commencer la représentation, les spectateurs sont reçus à l'un des accès du parc, situé près d'un petit potager communautaire. Nzaje, l'acteur angolais dont nous avons esquissé le profil dans la partie précédente, se mêle alors au public et déclame en boucle quelques vers d'un rap dont il est lui-même l'auteur :

Aujourd'hui, les poètes sont des héros

Ils changent des vies avec leurs mots

À présent mon peuple aura voix au chapitre 
Et nos mots ne seront pas escamotés ${ }^{11}$

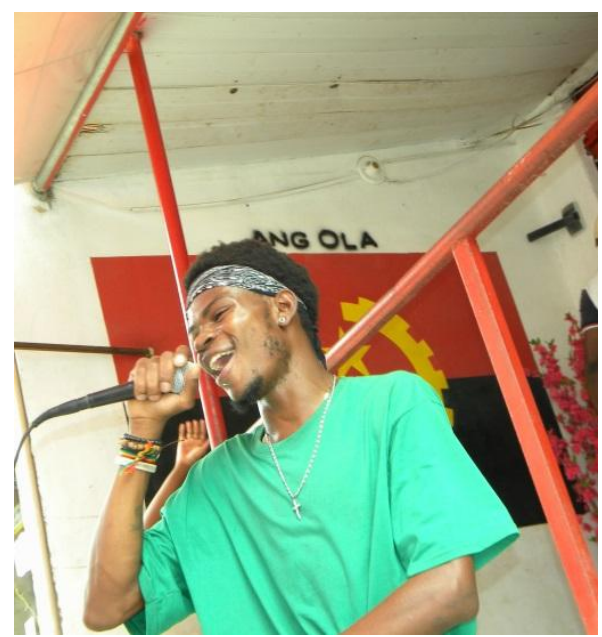

Figure 4 : Représentation musicale de Nzaje à Maré (Photo de l'auteur, 2019)

Cette introduction annonce une intention qui servira de fil rouge au spectacle : celle de rendre au sujet subalternisé une voix qui lui aurait été confisquée. Mais, on peut également comprendre dès à présent que les récits de vie qui seront présentés s'inscrivent dans le cadre d'un exercice poétique - et donc sans doute en partie imaginaire. Un autre jeune acteur d'origine angolaise se joint ensuite au public et l'enjoint à emprunter avec lui un chemin escarpé. Durant ce parcours, il narre brièvement "l'amour interdit" qui unit ses parents et les obligea à fuir de Luanda pour le Brésil, pays où lui-même est né. Une fois au sommet du petit morne, une actrice, fastueusement vêtue, surgit d'entre les arbres et se présente au public comme la reine africaine Nzinga Mbandi ${ }^{12}$. Elle s'exprime tour à tour en lingala ${ }^{13}$ et dans un portugais légèrement teinté $d$ 'accent francophone ${ }^{14}$. "Ma maison était ravissante, mais la guerre l'a détruite", explique ce nouveau personnage, avant d'évoquer son mariage forcé, puis la fuite et une longue errance de par le monde, pour finalement trouver refuge au Brésil, dans la favela : “Je rame désormais à Maré, j'ai ici ma famille, mes amis, mon histoire”,

\footnotetext{
${ }^{11}$ De même que ceux qui suivront, ce fragment de texte a été traduit librement à partir de l'original en portugais. Je profite de l'occasion pour remercier la directrice, Isabel Penoni, de m'avoir transmis le script complet de la pièce.

${ }^{12}$ Personnage historique, qui dirigea le Royaume de Ndongo (correspondant à l'actuel Angola) au XVII ${ }^{\mathrm{e}}$ siècle. En dépit des controverses historiques autour des ses actions, la figure de Nzinga est aujourd'hui très souvent mobilisée comme symbole de la puissance de la femme africaine et de résistance à la colonisation.

${ }^{13}$ Le lingala est l'une des langues bantoues parlées dans le nord de l'Angola, en République centrafricaine et, surtout, dans les deux Congo.

${ }^{14}$ La persistance de cet accent est compréhensible au vu de l'itinéraire biographique de l'actrice, Ruth Mariana. Si elle affirme être née angolaise, celle-ci a en effet passé une grande partie de sa jeunesse en République Démocratique du Congo, avant de revenir vers l'Angola pour échapper à de violents conflits, et finalement émigrer au Brésil.
} 
déclare-t-elle. S'ensuit un parcours à travers une partie verdoyante du parc. La reine examine plusieurs plantes et feuilles, en les désignant par leurs noms africains et en soulignant leurs vertus curatives ou encore culinaires. Le personnage s'enquiert auprès du public de l'usage qui est fait de ces mêmes espèces au Brésil. Le parcours botanique offert par le personnage de la pièce de théâtre relève donc d'une volonté de rappeler les liens qui unissent les deux continents, en soulignant la valeur épistémologique d'une culture africaine souvent peu valorisée au Brésil ${ }^{15}$.

La scène suivante nous emmène sur la partie la plus haute du parc, où s'ouvrent des points de vue spectaculaires sur la ville alentour. L'ensemble des membres brésiliens et angolais de la compagnie est à présent rassemblé pour ce qui est présenté comme une "festa na laje" ("fête sur la dalle")". Une ambiance surchauffée est installée par les acteurs, qui retirent une partie de leurs costumes et se trémoussent tandis que des percussions résonnent, entrainant les spectateurs.

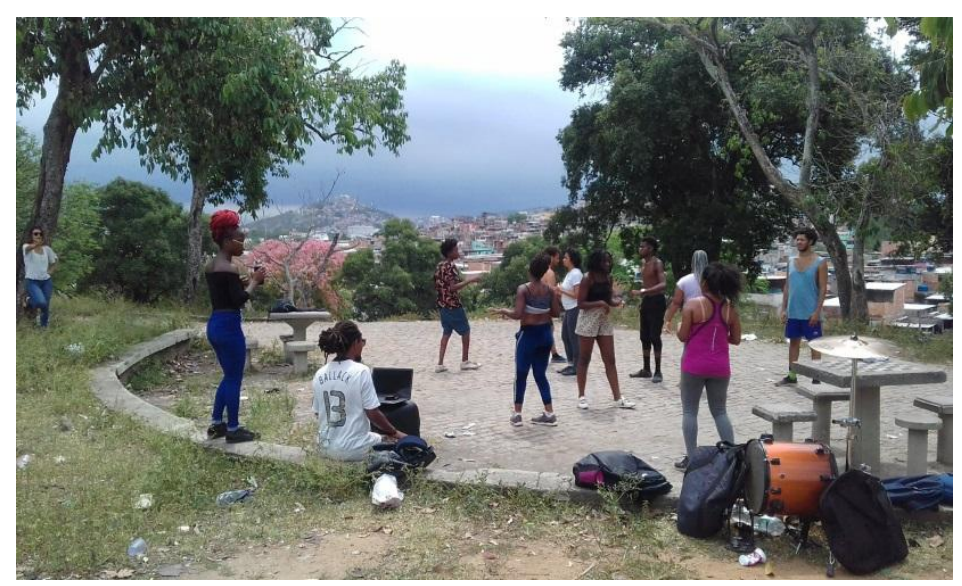

Figura 5 : Répétitions de la pièce au sommet du Parque Ecológico de Maré (Photo de l'auteur, 2019)

\footnotetext{
15 Dans un article de divulgation très stimulant, Alencastro (2019) a montré "l'ubiquité du métissage alimentaire" occasionné par des siècles d'échanges entre "pratiques culinaires africaines et afroaméricaines". Au cours du processus colonial, certaines plantes, fruits, légumes et féculents se transformèrent en "cultures véritablement pantropicales". L'historien souligne notamment que "le savoir ethnoécologique et les préférences alimentaires des Africains se révélèrent déterminants pour l'élaboration des cultures et des préparations parmi les communautés coloniales et nationales américaines". Comme le signale l'auteur, cet héritage africain a néanmoins souvent été éludé par les manuels de cuisine de référence, alors que le nom même de certains plats brésiliens et d'ingrédients très habituels révèle leur origine transatlantique.

${ }^{16}$ Dans les favelas de Rio de Janeiro, les dalles-terrasses des demeures autoconstruites sont des espaces emblématiques, dotés d'importantes fonctions pratiques (stockage de matériel, séchage des vêtements, etc.), mais souvent également destinés à un usage récréatif. En outre, de par son caractère privatif, la laje est un élément qui contribue fortement à la différenciation résidentielle au sein même des quartiers défavorisés : "Elles sont un patrimoine valorisé, un symbole de statut, un privilège qui fait des envieux" (Freire-Medeiros $2010: 6$ ).
} 
À Maré, les nombreux évènements festifs qui se produisent — souvent en pleine rue - constituent autant d'occasions susceptibles de rassembler Brésiliens et Angolais. Au-delà des différences culturelles dont on se plaint parfois, un certain hédonisme et un goût pour les relations chaleureuses semblent être partagés par beaucoup d'entre eux. Si l'on en croit Bruno, Angolais de 34 ans qui vit à Maré depuis 2012 :

"Les gens de Rio ressemblent à ceux de Luanda. Tout le monde aime la fête, les bons moments, une petite bière. (...) Ce sont ces aspects qu'on a en commun. Tu arrives quelque part sans connaitre personne, et tu sors de là avec un million d'amis."

Dans la représentation théâtrale que nous analysons, si la dalle sur laquelle la fête est censée battre son plein n'est qu'imaginaire, la position en surplomb depuis le point culminant du parc écologique permet effectivement à tous d'observer l'espace urbain qui se déploie plus bas. Les spectateurs sont incités à chercher dans quelle direction se trouve le centre-ville, situé à une dizaine de kilomètres. Mais une des actrices oppose alors que, pour elle, le centre de son existence se trouve juste en contrebas, dans la favela. Plus particulièrement sur une dalle où se trouve une citerne d'eau bleue dans laquelle elle entrait lorsqu'elle était enfant, pour se baigner lorsque la chaleur se faisait par trop accablante. Le positionnement militant qui motive les créations de la compagnie est de nouveau manifeste : il s'agit de replacer la périphérie au centre de l'attention, ainsi que de conférer une légitimité à des pratiques populaires très courantes, tel le détournement d'une réserve d'eau domestique pour en faire une piscine improvisée.

L'une des sources d'inspiration revendiquées par Cia Marginal est le "Teatro do Oprimido" (Théâtre de l'Opprimé). Cette expérience éminemment politique, menée à partir des années 1960 par Augusto Boal, servit à l'élaboration d'une méthode dramaturgique encore appliquée aujourd'hui par plusieurs groupes dans le monde ${ }^{17}$. Or, pour le dramaturge et militant carioca, "il ne suffit pas que le spectateur réfléchisse, il faut aussi qu'il agisse, qu'il se déplace, qu'il fasse” (Boal, apud Pereira 1998). Dans une tentative de mise en pratique de ces principes, à l'issue de la scène précédente, les acteurs entrainent le public pour un second mouvement vers un autre point du parc. Un

\footnotetext{
${ }^{17}$ Il existe notamment un Théâtre le l'opprimé en activité à Paris : 〈http://www.theatredelopprime.com> Consulté le 18/11/2020
} 
dispositif ludique est alors mis en place dans le cadre duquel la participation des spectateurs est sollicitée. Le "jeu" commence par des propositions très anodines, telles : "qui est déjà monté au sommet de la Pierre de Gávea ?"18. Les personnes qui se reconnaissent dans cette phrase doivent alors défiler au milieu de l'allée, au rythme d'un tambour énergique et de chants entrainants. Mais l'ambiance frivole se tend progressivement lorsque des questions plus dérangeantes commencent à être posées par les acteurs, se rapportant principalement aux discriminations structurelles qui persistent au sein de la société brésilienne. "Qui a déjà rangé son téléphone portable en apercevant un Noir dans la rue ?", est-il par exemple demandé au public-participant, alors qu'une atmosphère inconfortable s'installe. D'autres questions lancées achèveront de transformer le jeu en un moment de réflexion collective autour du racisme et de sa négation dans le contexte brésilien.

Porter sur la place publique la nécessité de débattre et d'adopter des mesures afin de lutter contre les discriminations basées sur la couleur de la peau ne s'est généralement pas fait sans frictions au Brésil. En effet, le récit national en vigueur depuis la première moitié $\mathrm{du} \mathrm{XX}^{\mathrm{e}}$ siècle alléguait la quasi-absence de préjugé racial. L'influence des travaux de Gilberto Freyre (2005) sur la formation de ce mythe moderne de la brésilianité est bien connue. L'idée d'un pays génétiquement et culturellement métissé, fruit de la promiscuité ancienne entre maîtres blancs et esclaves noirs se diffusa très largement dans la société à partir des années 1930. Le régime réformiste et autoritaire de Getúlio Vargas se saisit de la vision sociale suggérée par l'œuvre de Freyre (associée au concept postérieurement créé de "démocratie raciale") pour en faire "une sorte d'idéologie non officielle de l'État, permettant de couvrir les clivages de race et de classe ainsi que des conflits sociaux qui s'accumulaient à l'époque" (Schwarcz 1993 : 325). En contraste notamment avec les États-Unis, où la ségrégation régissait de manière stricte les relations entre groupes ethniques antagoniques, le Brésil forgea ainsi de lui-même une représentation a priori plus positive, caractérisée par un prétendu talent à réunir et à amalgamer les différentes races de façon tendanciellement harmonieuse. Néanmoins, dès les années 1950, le récit presque idyllique des relations raciales brésiliennes fut mis en doute par des intellectuels nationaux et étrangers qui se penchaient empiriquement sur cette question (Maio 1999). En parallèle, des organisations militantes n'eurent de cesse de dénoncer

\footnotetext{
${ }^{18}$ Accident géographique situé au bord de la mer, dans la zone sud de la ville.
} 
avec vigueur la place systématiquement subalterne des Noirs dans la société, formulant des revendications afin de compenser l'absence de mesures en faveur de l'intégration des descendants d'esclaves après l'abolition — tardive — de 1888 (Nascimento 1978). Ces dernières décennies, au diapason des débats qui agitent l'Amérique latine, les questions liées à l'ethnie et à la race ont acquis une importance croissante au Brésil ; devenant une importante clé d'interprétation du conflit social (Verdo \& Vidal 2012). En dépit de mesures très commentées concernant notamment l'action affirmative (Santos 2018), le volontarisme politique visant à traiter ce problème reste faible.

\section{Un dialogue qui voit se confronter les imaginaires sud-atlantiques}

Cette contextualisation concernant la première partie de la pièce s'avère utile pour mieux appréhender une scène ultérieure — dite de la "Bataille" — qui a plus particulièrement retenu mon attention. Au cours de celle-ci, les acteurs se répartissent en deux groupes : d'un côté les Brésiliens; de l'autre les Angolais. En mettant en exergue par la caricature les principaux malentendus culturels qui naissent de leur cohabitation au sein de la favela, l'échange qui s'ensuit est censé révéler les représentations et préjugés construits par un groupe sur l'autre.

Une comédienne angolaise déclare ainsi d'emblée qu'avant de venir au Brésil, elle pensait trouver en ces terres uniquement "des Blancs, de grands immeubles et une ville propre". Cette vision pourrait sembler surprenante à un spectateur européen, généralement habitué à des images qui privilégient les "lieux et paysages de désenchantement et de peur" (Ulhôa 2017 : 13) lorsqu'il s'agit de représenter l'espace urbain brésilien. La réplique correspond néanmoins fidèlement aux déclarations d'une grande partie des Angolais rencontrés à Maré quand ils évoquent leur imaginaire préalable à la migration. Lorsque j'interrogeais mes interlocuteurs à propos de l'idée qu'ils se faisaient du Brésil avant leur arrivée, le même type de réponses était généralement obtenu. Par exemple, pour Carlos, 43 ans, originaire de Luanda et qui vit à Rio de Janeiro depuis 1997,

\footnotetext{
"L'image que nous avions c'était surtout à travers des telenovelas $^{19}$. Les novelas, elles entraient chez nous, et elles entrent toujours. Alors, la première fois, quand je suis arrivé ici, je
}

\footnotetext{
${ }^{19}$ Feuilletons télévisés.
} 
me disais "je vais vivre à Copacabana, je vais sortir avec ces Brésiliennes blanches, aux yeux verts et avec une longue chevelure". C'était ça, l'image."

L'ensemble des témoignages recueillis, que ce soit lors d'interviews ou de conversations informelles, ne laisse aucun doute sur le fait qu'en Angola les "telenovelas" brésiliennes sont suivies et appréciées par un large public ${ }^{20}$. Si l'on en croit le discours généralement mobilisé, le Brésil imaginé par les Angolais serait incontestablement celui promu par l'industrie du divertissement de masse. Or, cette "usine à rêves" utilise régulièrement un Rio de Janeiro fortement idéalisé et glamourisé pour la mise en scène de ses productions audiovisuelles. Un fait surprenant découle de l'influence des "novelas" sur les représentations des Angolais: mes interlocuteurs affirmèrent souvent qu'ils ignoraient purement et simplement l'existence des favelas avant leur arrivée au Brésil. Helena, femme angolaise de 47 ans, venue pour la première fois à Rio en 2011 fut d'ailleurs catégorique dans ce sens :

"Je me disais que le Brésil était un pays où il n'y aurait pas, par exemple, des favelas comme celle que je vois ici. Je pensais que c'était de partout comme à Copacabana. On regarde les novelas brésiliennes, alors je me suis inspirée de cela. C'est seulement en venant ici que j'ai vu la réalité. En Angola, je vivais déjà dans une favela, qu'on appelle “musseque”, alors, mon espoir c'était que le Brésil soit un pays tout joli, bien organisé, sans bazar de partout, sans favelas."

Mais, dans la pièce de théâtre, si l'ingénuité apparente du personnage angolais provoque immédiatement les sarcasmes des brésiliens, le jeu des stéréotypes suit une construction en miroir. Ainsi, s'adressant au public, un comédien brésilien se prend à rêver d'un voyage en Angola à l'arrivée duquel "une foule de gens noirs d'ébène ${ }^{21 \text { ", }}$ serait là pour l'accueillir, en lui souhaitant la bienvenue comme à un "frère". C'est alors qu'un Angolais l'interrompt, pour lui signaler que là-bas, il serait plutôt considéré

\footnotetext{
${ }^{20}$ On pourra également mentionner une information aussi insolite qu'édifiante : à Sambizanga, en périphérie de Luanda, un complexe commercial informel, souvent considéré comme le plus grand marché à ciel ouvert d'Afrique s'était vu attribuer populairement le nom de "Roque Santeiro", qui n'est autre que le nom d'une série à succès de la chaîne Rede Globo diffusée au milieu des années 1980. Ce marché populaire a été fermé par les pouvoirs publics angolais en 2011.

${ }^{21}$ Le personnage utilise l'expression "pretos retintos", actuellement utilisée au Brésil pour désigner les Noirs dotés d'une peau plus sombre.
} 
comme un Blanc, et pas nécessairement comme un "frère". Cette allégation suscite son ire. "Dans le meilleur des cas, tu serais vu comme un 'mulato' ('mulâtre')", nuance un autre Angolais. Le Brésilien, doté d'une chevelure crépue, mais d'un teint de peau relativement clair, s'en agace encore davantage : “Mulato' ici, c'est le petit de la mule, en Angola ça veut dire quoi ?"; "C'est un synonyme de riche" décoche alors une Angolaise, provoquant les rires de tous.

Cette fraction du dialogue met en évidence un entrecroisement de problématiques qu'il est intéressant de démêler. On pourra tout d'abord se pencher sur une certaine vision de l'Afrique - suggérée par le personnage brésilien lorsqu'il se prend à rêver de fraternités transatlantiques. Dans l'acceptation courante du terme, le panafricanisme considère comme membres d'une diaspora les descendants d'Africains à divers degrés vivants hors du continent ${ }^{22}$. Cette communauté transnationale serait basée sur une lignée, mais surtout sur un patrimoine culturel fortement hybride (Gilroy 2003). Le projet de réunion de l'Afrique avec sa diaspora a souvent été porté par des intellectuels (tels W. E. B. Du Bois) ou encore des activistes et entrepreneurs (par exemple, Marcus Garvey) basés aux États-Unis. Pour les Afro-Brésiliens, l'élaboration d'une identité collective valorisante est également souvent passée par un rapprochement affectif, religieux, esthétique, artistique ou même politique avec l'Afrique. Des dynamiques de réafricanisation ont ainsi pu été observées à travers une quête de “pureté" du rite religieux dans la pratique du candomblé (Capone 2000), ou encore lors de l'apparition de formations carnavalesques mues par une affirmation identitaire (Agier 2000). Néanmoins, jusqu'à peu, l'incitation à tourner le regard vers l'Afrique en vue d'une régénération culturelle, psychologique ou spirituelle s'est la plupart du temps limitée à des cercles restreints de personnes, généralement de niveau socio-économique plutôt élevé.

Pour Paul Gilroy, il existerait le risque d'une approche biaisée de l'Afrique de la part de certains Américains en quête de leurs origines. Cette situation pourrait aboutir à la formulation d'une "africanité fortement mythologisée, qui elle-même trouve son origine non pas en Afrique, mais dans une variété de l'idéologie panafricaniste produite tout récemment par l'Amérique noire" (Gilroy 2003 : 123). Or, l'idéal panafricain se

\footnotetext{
${ }^{22}$ L'Union Africaine reconnait d'ailleurs officiellement "le rôle important que doit jouer la diaspora africaine dans le développement du continent" $<\underline{\text { https://au.int/fr/implication-diaspora-societe-civile }>}$ Consulté le 21/09/2020.
} 
heurte depuis ses origines non seulement au souverainisme réfractaire de certains dirigeants, mais également aux perceptions variées (ou encore à l'indifférence) des Africains continentaux les uns envers les autres, et plus encore envers les membres de la “diaspora”. Dans le cas qui nous intéresse, le livre de Joana Neitsch et Juliana Passos (2010) sur le quotidien de Brésiliens à Luanda a notamment montré l'asymétrie qui caractérise certaines relations entre expatriés (même noirs) et locaux en Angola. La tendance émergente du Brésil à exercer un "sous-impérialisme" économique en Afrique lusophone (Ridderbusch 2018) peut également parfois être source de tensions.

Par ailleurs, en raison du processus colonial, l'élite luandaise était traditionnellement constituée de nombreux métis (Santos Nascimentos 2018). Après l'indépendance de 1975, certains sont restés proches des strates du pouvoir (MabekoTali 2001) et ont gardé une certaine mainmise sur l'économie ou encore le secteur culturel. En contraste, les couches paupérisées des grandes villes et de l'arrière-pays sont presque hégémoniquement constituées d'individus à la peau foncée. Ainsi, même si démographiquement la population blanche est très minoritaire aujourd'hui en Angola, une peau légèrement plus claire (comparable à celle de nombreux Brésiliens qui se considèrent comme "Noirs") est généralement associée à une position privilégiée dans la société. Cela explique l'équivalence faite par une comédienne entre "mulâtre" et "riche".

$\mathrm{Au}$ terme de la scène de la "Bataille", Angolais et Brésiliens se querellent d'ailleurs une dernière fois au sujet de la colonisation de leur pays respectif par le Portugal. Ils finissent toutefois par développer une complicité autour de certains vocables courants au Brésil, mais dont l'étymologie renvoie aux diverses langues parlées traditionnellement en Angola (p. ex. "berimbau”, "dendê", "cafuné”, "quilombo", "senzala”, “capanga”, etc.). La confrontation prend alors un tour musical et chorégraphique : les acteurs se mélangent et dansent au rythme d'un "funk carioca",23 avant d'enchainer sur des mouvements saccadés de "kuduro",24. Un nouveau morceau est alors diffusé et repris par tous (y compris par le public, invité à rejoindre la célébration) : il s'agit de la chanson "Hoje não saio daqui", de la formation angolaise Gang Machado, qui a donné son titre au spectacle.

\footnotetext{
${ }^{23}$ Terme générique désignant une musique électronique typique des favelas, caractérisée par des motifs rythmiques spécifiques ("pancadão", "tamborzão" ou "batidão")

${ }^{24}$ Style de musique électronique angolaise, généralement accompagnée de chorégraphies énergiques.
} 
En visionnant le vidéo-clip qui accompagna la sortie du morceau ${ }^{25}$, il apparaît sans équivoque possible que le message délivré par ce tube afro-house de 2013 se rapporte à l'ambiance débridée des clubs de nuit (“Aujourd'hui, je ne partirai pas d'ici, je ne m'en irai que demain matin" scandent en boucle les interprètes). Or, ce texte léger évoque une correspondance paradoxale avec les mots d'une chanson brésilienne bien connue: “Opinião”, de Zé Keti. Les paroles de cette composition, lancée en 1964, opposaient une fin de non-recevoir aux menaces d'expulsion que le gouvernement faisent alors peser sur les habitants de nombreuses favelas à Rio de Janeiro. "Vous pouvez m'arrêter, vous pouvez me frapper, vous pouvez me laisser sans rien à manger, ça ne me fera pas changer d'idée" fredonne l'interprète Nara Leão sur l'enregistrement original $^{26}$, avant de conclure effrontément : "Daqui do morro eu não saio não" ("Je ne partirai pas de la favela").

"Hoje não saio daqui" et "Daqui do morro eu não saio não" : le rapprochement entre ces deux refrains - l'un marqué par l'hédonisme des couche-tard ; l'autre par une posture de résistance farouche - paraît de prime abord un contre sens absolu. Néanmoins, un lien improbable est établi entre eux par une réplique de la scène finale, lorsqu'une actrice brésilienne proclame, au sujet de la favela dans laquelle elle vit :

“Ici, il n'y avait que la forêt. Il n'y avait pas d'électricité, ni de gens. J'aime cet endroit (...) On a essayé tant de fois de m'en chasser, de m'expulser, de me tuer. Mais, ça ne sert à rien d'insister, de m'envahir, de me faire de fausses promesses, parce qu'aujourd'hui, je ne partirai pas d'ici (“hoje não saio daqui”).”

La transfiguration d'un refrain festif en un cri de ralliement contre l'oppression dont sont victimes les habitants des favelas est ainsi accomplie, traçant une continuité entre pratiques récréatives et culture politique.

\section{Conclusions}

Le dialogue sud-atlantique entre Angola et Brésil semble fait depuis des siècles de rapprochements et d'éloignements successifs, tout en étant parsemé de nombreux malentendus. Comme l'a évoqué Alencastro (2020), certains éléments laissent penser

\footnotetext{
$25<$ https://youtu.be/W_SfmhsHbU> Consulté le 21/09/2020

${ }^{26}<$ https://www.youtube.com/watch?v=yxvjtMYKORg > Consulté le 21/09/2020
} 
que "le $\mathrm{XXI}^{\mathrm{e}}$ siècle assiste à une nouvelle rencontre entre les deux rives de l'Atlantique Sud". Ce phénomène serait favorisé, d'après l'auteur, par l'exportation d'églises évangéliques ainsi que de produits culturels brésiliens de l'autre côté de l'océan ; mais également par l'émergence d'un courant migratoire entre l'Afrique subsaharienne et le Cône Sud de l'Amérique. Les termes de l'échange semblent néanmoins inégaux. À ce propos, 1'écrivain angolais Pepetela déplorait au cours d'une interview ${ }^{27}$ accordée à la presse le fait qu'il existe une profonde asymétrie dans la relation d'estime entre Brésil et Angola : "Les Angolais regardent vers le Brésil, mais les Brésiliens en général ignorent l'Angola".

L'implantation d'Angolais depuis plus de vingt ans à Rio de Janeiro s'inscrit ainsi dans un cadre complexe, qui tend souvent à jouer en leur défaveur. Aujourd'hui encore, l'intégration de ces étrangers à la société brésilienne demeure précaire. Plus particulièrement encore, les individus résidents à Maré se trouvent au centre de dynamiques croisées de discrimination — puisqu'étant étrangers, noirs, généralement assez pauvres et habitants d'un espace symboliquement et physiquement dégradé. En outre, les relations de ces immigrés avec les autres habitants de la favela sont parfois marquées par des tensions et des incompréhensions mutuelles.

Le spectacle "Hoje não saio daqui", de la Cia Marginal, représente une tentative de mise en récit de cette présence angolaise au sein des favelas de Maré. Le dispositif dramaturgique de l'œuvre repose très largement sur le fait que les acteurs angolais (ou descendants directs d'Angolais) accomplissent une performance visant à correspondre (pour mieux répondre) à certains préjugés et idéalisations qui se manifestent lors des interactions avec leurs voisins brésiliens. Leur participation permet ainsi de mettre en évidence la dimension situationnelle et socialement construite de l'altérité ethnique (Barth 1995).

27 〈https://www.bbc.com/portuguese/noticias/2011/09/110908_angola_entrevista_jc〉 Consulté le $19 / 11 / 2020$ 


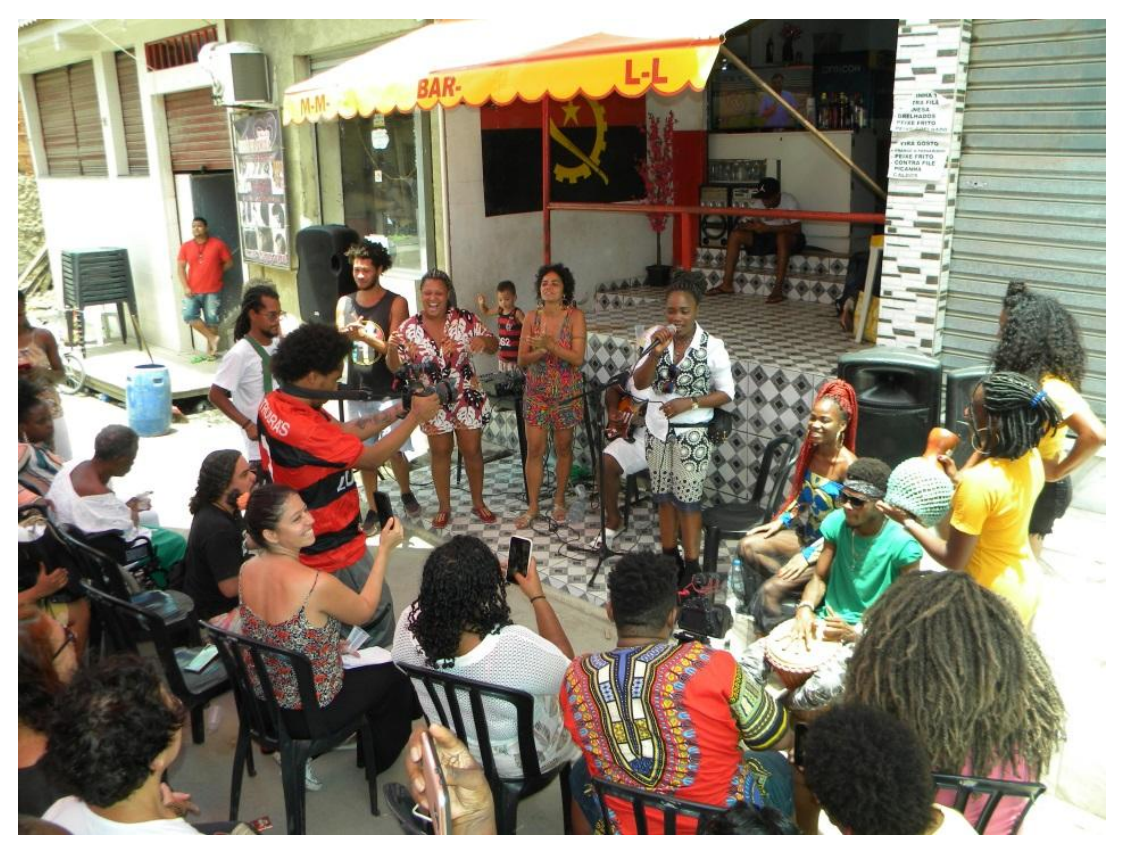

Figure 6 : Fête de lancement de la pièce devant un restaurant angolais de Maré (Photo de l'auteur, 2019)

La pièce suggère en outre qu'une nouvelle étape de la relation entre Angola et Brésil puisse être en train de se dérouler dans ce quartier périphérique symboliquement dégradé de Rio de Janeiro. La réunion d'acteurs/créateurs brésiliens et angolais au sein d'une troupe théâtrale montre qu'un dialogue peut se construire sur des bases horizontales. En commun entre les deux groupes, comme le montre le spectacle, on retrouve un goût prononcé pour certaines réjouissances qui réunissent fréquemment les habitants de la favela. Mais, la création théâtrale se saisit aussi de l'expérience angolaise afin de rendre particulièrement visible la question du racisme qui touche l'ensemble des personnes perçues, à des degrés divers, comme noires au Brésil. À ce titre, pour les Brésiliens qui résident dans la favela comme pour les immigrés Angolais, il est également possible de penser la nécessité d'une mobilisation face à l'hostilité d'une partie de la société et à ses logiques d'exclusion. Si cette résistance n'est pas toujours réalisée de façon conjointe, elle permet tout du moins d'imaginer des passerelles à créer entre différents. Les enquêtes de terrain confirment l'intensité des interactions qui se sont développées entre Brésiliens et Angolais au sein de l'espace caractéristique des favelas de Maré. Il s'agit d'une relation complexe, faite de conflits aussi bien que de convivialités. 


\section{Références bibliographiques}

Agier, M. 2000, Anthropologie du Carnaval. La ville, la fête et l'Afrique à Bahia, Paris, Éditions Parenthèses.

Alencastro, L. F. 2020, “L’Atlantique Sud”, Portail Transatlantic Cultures. consulté le 21/09/2020 <https://www.transatlantic-cultures.org/fr/catalog/l-atlantique-sud >

Alencastro, L. F. 2019, “Viagens culinárias entre a África e a América”, Revista Quatro $\begin{array}{lllll}\text { Cinco } U m \text {. Consulté } & 18 & \text { décembre }\end{array}$ 2020. <https://quatrocincoum.folha.uol.com.br/br/artigos/historia/viagens-culinariasentre-a-africa-e-a-america>

Alencastro, L. F. 1986, Le commerce des vivants : traite d'esclaves et Pax Lusitana dans l'Atlantique Sud, XVIe siecle-XIXe siecle, These de Doctorat en Histoire, Université de Paris-X - Nanterre.

Aydos, M. R. 2010, "Migração forçada: uma abordagem conceitual a partir da imigração de angolanos para os estados do Rio de Janeiro e São Paulo, Brasil (19702006)", Mémoire (mestrado) - Universidade Estadual de Campinas, Instituto de Filosofia e Ciências Humanas.

Barth, F. 1995, Les groupes ethniques et leurs frontières, in P. Poutignat \& J. StreiffFénart eds, Théories de l'ethnicité, Paris, PUF : 203-249.

Capone, S. 2000, “Entre Yoruba et Bantou”, Cahiers d'études africaines, 157 : 55-78.

Cahen, M \& Dos Santos, I. 2018, “Lusotopie, Lusotopy. What Legacy, What Future?”, Lusotopie, XVII (2): 187-203.

Diniz, E. 2013, Memória e identidade dos moradores do Morro do Timbau e Parque Proletário da Maré, Rio de Janeiro, Redes da Maré.

Freire-Medeiros, B. 2010, "Casa, Rua \& Laje: O caso da favela globalizada", paper présenté durant le Seminário Internacional de Sociologia: 50 anos de Brasília e 40 anos da Pós-Graduação do Departamento de Sociologia da Universidade de Brasília - UnB, 9 a 12 de novembro de 2010. 
Freyre, G. 2005, Casa-Grande \& Senzala, 50ª edição, Rio de Janeiro, Global Editora [1933].

Gilroy, P. 2003, L'Atlantique noir. Modernité et double conscience, Paris, Kargo.

Maio, M. C. 1999, "O Projeto Unesco e a agenda das ciências sociais no Brasil dos anos 40 e 50", Revista Brasileira de Ciências Sociais, 14 (41): 141-158.

Jacques, P. B. \& Varella, D. \& Bertazzo, eds. 2002, Maré: Vida na Favela, Rio de Janeiro, Casa da Palavra.

Leite, B. Paixão. 2013, “Com organização e luta construindo a Maré que queremos: experiências de mobilização popular em Nova Holanda", Monografia de Especialização, Rio de Janeiro, UFRJ, IPPUR.

Mabeko-Tali, J.-M. 2001, Dissidências e poder de estado: o MPLA perante si próprio (1962-1977): ensato de história politica, Luanda, Nzila.

Muñoz, E. E. Ed. 2016, "Dossiê: A cooperação Sul-Sul do Brasil com a África", Caderno CRH, 29 (76), Salvador, UFBA.

Nascimento, A. 1978, O genocídio do negro brasileiro: processo de um racismo mascarado, Rio de Janeiro, Paz e Terra.

Neitsch J.\& Passos, J. 2010, "Fixe malaike: o cotidiano dos brasileiros em Angola", Departamento de Jornalismo, Universidade Federal de Santa Catarina.

Petrus, M. R. 2001, Emigrar de Angola e imigrar no Brasil: jovens imigrantes angolanos no Rio de Janeiro: história(s), trajetórias e redes sociais, Mémoire (Mestrado), Rio de Janeiro, IPPUR/UFRJ.

Pereira, A. 1998, “A Poética do Oprimido e o papel do espectador no jogo e debate teatrais", Caravelle, 70: 151-164.

Quirion, N. 2018, “Um triplo grau de alteridade: representações midiaticas sobre migrantes da África Central nas favelas do Rio de Janeiro", Anais do III Urbfavelas Salvador, 21 a 23 de novembro de 2018, Salvador, UCSal.

Redes da Maré eds. 2018, Censo Populacional da Maré, Rio de Janeiro, Redes da Maré. 
Ridderbusch, J. 2018, Cooperação Sul-Sul entre o Brasil e a África subsaariana: A política externa brasileira em Angola e Moçambique, Mémoire (Mestrado) em Estudos Latino-americanos, Políticas Públicas, Leiden, Universidade de Leiden.

Santana, J. A. C. 2016, Imigrantes africanos em um Conjunto de favelas no Rio de Janeiro: redes sociais, disputas, trabalho informal e ilegalismo. Mémoire (mestrado) Instituto de Filosofia e Ciências Humanas, Rio de Janeiro, UFRJ.

Santos Nascimento, W. 2018, "Trânsitos, pertencimentos e resistências da elite mestiça de Luanda, Angola (1950 - 1980)”, Revista Territórios e Fronteiras, XXI (1): 341-357.

Santos, R. E. N. D. 2018, “Ações Afirmativas no combate ao racismo: uma análise da recente experiência brasileira de promoção políticas públicas”, Revista Quaestio Iuris, v.11 : 2101-2128.

Schwarcz, L. M. 1993, O Espetáculo das Raças - cientistas, instituições e questão racial no Brasil 1870-1930, São Paulo, Companhia das Letras.

Ulhoa, L. M. 2017, “Les images des villes brésiliennes dans les manuels scolaires de France et du Brésil : entre mythes et réalités”, Confins 33, consulté le 21/09/2020. http://journals.openedition.org/confins/12434.

Vaz, L. F. 2010, “Um território híbrido na Maré, RJ. Novo território cultural?”, communication présentée lors du séminaire international Política cultural: teoria $e$ práxis, 22-24 septembre 2010, Rio de Janeiro, Fundação Casa Rui Barbosa.

Verdo, G. \& Vidal, D. 2012, "L'ethnicité en Amérique latine : un approfondissement du répertoire démocratique ?", Critique internationale, 4 (57) : 9-22. 